\title{
Cyclic Strain Mitigates Nanoparticle Internalization by Vascular Smooth Muscle Cells
}

\author{
Chia-Liang Tsai' , Ching-Yun Huang ${ }^{2}$, Yi-Ching Lu', Li-Mei Pai $\mathbb{D}^{3,4}$, Daniel Horák $\mathbb{D}^{5}$, Yunn-Hwa Ma $\mathbb{D}^{1,6}$ \\ 'Department of Physiology and Pharmacology, Chang Gung University, Taoyuan, 33302, Taiwan, Republic of China; ${ }^{2}$ Institute of Biomedical Sciences, \\ Chang Gung University, Taoyuan, 33302, Taiwan, Republic of China; ${ }^{3}$ Department of Biochemistry \& Molecular Biology, College of Medicine, Chang \\ Gung University, Taoyuan, 33302, Taiwan, Republic of China; ' ${ }^{4}$ Liver Research Center, Chang Gung Memorial Hospital, Taoyuan, 33305, Taiwan, \\ Republic of China; ${ }^{5}$ Institute of Macromolecular Chemistry, Academy of Sciences of the Czech Republic, Prague 6, 162 06, Czech Republic; \\ ${ }^{6}$ Department of Medical Imaging and Intervention, Chang Gung Memorial Hospital, Taoyuan, 33305, Taiwan, Republic of China
}

Correspondence: Yunn-Hwa Ma, Department of Physiology and Pharmacology, Chang Gung University, Guishan, Taoyuan, 33302, Taiwan, Republic of China, Email yhma@mail.cgu.edu.tw

Background: Intravascular delivery of nanoparticles for theranostic application permits direct interaction of nanoparticles and vascular cells. Since vascular smooth muscle cells (VSMCs), the major components of the vascular wall, are constantly subjected to mechanical stimulation from hemodynamic influence, we asked whether cyclic strain may modulate internalization of magnetic nanoparticles (MNPs) by cultured VSMCs.

Methods: Cyclic strain (1 Hz and 10\%) was applied with Flexcell system in cultured VSMCs from rats, with cell-associated MNPs $\left(\mathrm{MNP}_{\text {cell }}\right)$ determined by a colorimetric iron assay. Transmission and scanning electron microscopy were used for morphology studies. Confocal microscopy was used to demonstrate distribution of actin assembly in VSMCs.

Results: Incubation of poly(acrylic acid) (PAA)-coated MNPs with VSMCs for $4 \mathrm{~h}$ induced microvilli formation and MNP internalization. Application of cyclic strain for 4-12 h significantly reduced $\mathrm{MNP}_{\text {cell }}$ by up to $65 \%(p<0.05)$, which was associated with blunted microvilli and reduced vesicle size/cell, but not vesicle numbers/cell. Confocal microscopy demonstrated that both cyclic strain and fibronectin coating of the culture plate reduced internalized MNPs, which were co-localized with vinculin. Furthermore, cytochalasin D reduced $\mathrm{MNP}_{\text {cell }}$, suggesting a role of actin polymerization in MNP uptake by VSMCs; however, a myosin II ATPase inhibitor, blebbistatin, exhibited no effect. Cyclic strain also attenuated uptake of PAA-MNPs by LN-229 cells and uptake of polyL-lysine-coated MNPs by VSMCs.

Conclusion: In such a dynamic milieu, cyclic strain may impede cellular internalization of nanocarriers, which spares the nanocarriers and augments their delivery to the target site in the lumen of vessels or outside of the circulatory system.

Keywords: cyclic strain, magnetic nanoparticles, endocytosis, actin

\section{Introduction}

Intravascular delivery has been employed as a major route of administration for delivery of nanocomposites in targeted therapy or diagnosis. Capacity of nanoparticle uptake by vascular cells has been demonstrated, which appears to be timeand concentration-dependent. ${ }^{1-3}$ Therefore, interaction of nanocomposites and the vascular wall may cause vascular retention and thus impact on the targeting effects and efficacy of the treatment.

Vascular smooth muscle cells (VSMCs) are the major component of the vascular structure, which are constantly subjected to mechanical forces including cyclic strain or tension created by the beating heart. Cyclic strain acts as an important modulator in cell morphology and function, including alignment, migration and proliferation, etc. ${ }^{4-7}$ Since VSMCs are the main component of the vessel wall and are responsible for the regulation of the vascular tone and systemic resistance, the response of VSMCs to mechanical stimuli from the pulsatile hemodynamics has been the focus of interest. ${ }^{8,9}$ When mechanical force changes the behavior of cells, two main processes must have occurred, i.e. mechanosensing and response to the force. ${ }^{10}$ Mechanosensing requires configuration changes of the mechanosensors, including channels, cytoskeletal filaments, and cytosolic/nuclear proteins. With one end of the mechanosensors connected 


\section{Graphical Abstract}

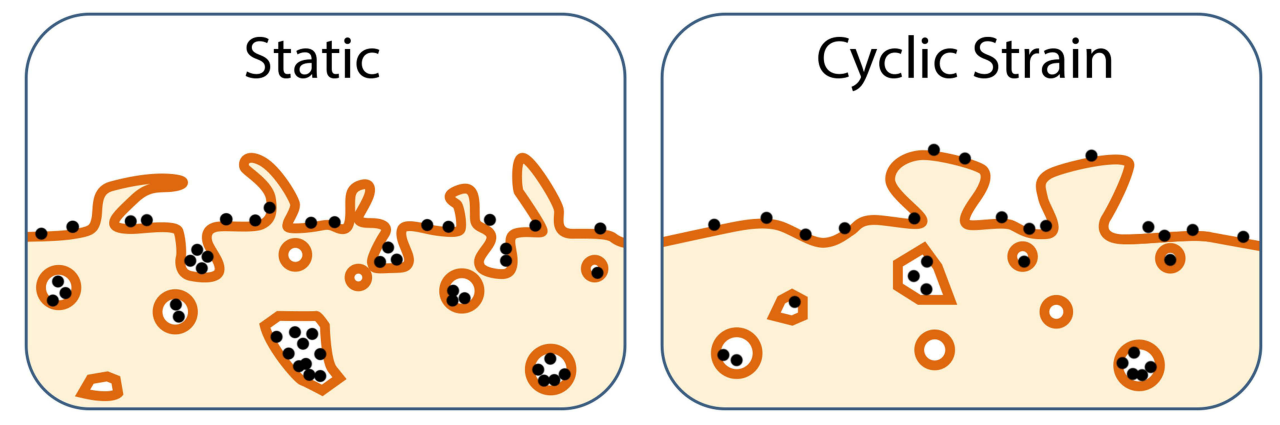

to the relatively fixed tissue, such as cytoskeletons or extracellular matrices, while the other end is pulled along the direction of the force, VSMCs may detect changes in the extracellular mechanical stimuli including tensile stress, cellular boundary, substrate stiffness and topography. In response to cyclic strain, VSMCs express stress fiber and transform from contractile to synthetic phenotype characterized by morphology, expression of marker proteins, and cellular physiology. ${ }^{11}$ Many signaling molecules and growth factors have been demonstrated to participate in cyclic strain-induced cellular events, ${ }^{12}$ suggesting a profound influence of cyclic strain on VSMC function. Although VSMCs may be exposed to nanoparticles administered for diagnostic or therapeutic application in inflammatory vascular diseases, ${ }^{13}$ whether cyclic strain may modulate nanoparticle uptake by VSMCs remains unknown.

Uptake of nanoparticles is mediated predominantly via endocytosis with a variety of mechanisms, including clathrinor caveolin-dependent endocytosis, phagocytosis, macropinocytosis, and receptor-mediated endocytosis etc. ${ }^{14}$ Nanoparticle endocytosis may be described as a two-step process, where the nanoparticles initially adhere to the cell membrane and subsequently are internalized by the cells via energy-dependent pathways, ${ }^{15-17}$ which are associated with induction of plasma membrane ruffling, closuring and perturbations. Macropinocytosis is an actin-regulated process that involves wrinkling and engulfing large amounts of extracellular fluid and particles through the plasma membrane. The wrinkles of the membrane take different shapes, and when closed, they form large organelles, macropinosomes; ${ }^{18}$ in the process, actin assembly plays an essential role in the engulfment process. Previous studies have demonstrated the involvement of actin cytoskeleton during endocytosis, although to varying degrees. ${ }^{19,20}$

In the endocytosis processes, actin cytoskeleton plays multiple roles in the formation of protrusions, remodeling of the cell surface, inward vesicle trafficking, and cell motility. ${ }^{21,22}$ In addition, the structure and the dynamics of actin cytoskeleton also respond to external stimuli such as extracellular mechanical forces. ${ }^{22}$ Actin filaments are semi-flexible polymers that organize into diverse architectures and participate in cellular function, including the formation of parallel bundles in filopodia, and formation of branched or crosslinked networks in lamellipodium. ${ }^{23}$ During the formation of filopodia, the cross-linking of actin filaments may be a critical step because individual long actin filaments may lack sufficient stiffness to push the cell membrane efficiently. ${ }^{24}$ Although filopodia may be composed of different molecules or components, long bundled actin filaments are common characteristics that can grow or bundle dynamically to intersect with various signaling machinery and support the function of filopodia. ${ }^{23}$

Actin filaments exhibit the function of a mechanosensor for tensile force applied to cells, ${ }^{25}$ which strengthens and generates stress fibers in a direction parallel to the applied force, allowing the cell to counteract the external tensile forces. Mechanical tension induces the formation of focal adhesion (FA) and recruits a variety of proteins to form FA complex, which serves as a structural link between cells and surrounding tissues, and plays an important role in exchanging mechanical force cues and regulatory signals. ${ }^{26} \mathrm{FA}$ is a dense molecular assembly connecting extracellular matrix through integrin receptors and actin stress fibers in the cell. ${ }^{26}$ Upon binding of talin, integrin recruits more 
members of FA, including vinculin, paxillin and FA kinase. In addition to the original role as a mechano-coupler, vinculin is also responsible for regulating mechanical stress. ${ }^{26}$ The physical pressure induces the exposure of vinculin-binding domain on the talin. Once vinculin binds to talin and becomes activated, it is connected to FA, which is regarded as a bidirectional conduit for "inside-out" and "outside-in" signaling. ${ }^{27}$ As a result, cells can reorganize the cytoskeleton structure to undergo the correct adjustments in response to pulsatile strain change.

Magnetic nanoparticles (MNPs), composed of magnetite $\left(\mathrm{Fe}_{3} \mathrm{O}_{4}\right)$ or meghamite $\left(\gamma-\mathrm{Fe}_{2} \mathrm{O}_{3}\right)$ cores, exert great potential to serve as drug carriers for targeted delivery due to the unique superparamagnetic properties, allowing MNPs to be guided by magnetic force against blood flow. ${ }^{28}$ In addition to drug delivery, MNPs span a wide range of applications, including magnetic resonance imaging and magnetic hyperthermia. ${ }^{28-30}$ In all applications, the interaction of cells and nanoparticles can be crucial for the distribution and delivery that determines the pharmacodynamic and pharmacokinetic consequences. Although characteristics of the coating materials have been shown to play a critical role in nanoparticlecell interaction, ${ }^{31,32}$ limited studies have demonstrated that cyclic strain modulates cellular uptake of nanoparticles. ${ }^{2,33}$ In this study, MNPs were used as a model system, primarily due to the wide application of MNPs and the reproducible assay system for iron. Since actin rearrangement is associated with both cyclic strain and endocytosis, we asked whether cyclic strain within physiological range may reduce MNP uptake by VSMCs. The results demonstrated a time-dependent inhibitory effect of cyclic strain on VSMC uptake of MNPs with a variety of coatings, which may be due to interference of actin assembly induced by cyclic strain.

\section{Materials and Methods}

\section{Materials}

Poly(acrylic acid)-coated MNPs (fluidMAG-PAS; $200 \mathrm{~nm}$, denoted as PAA-MNPs), green fluorescent PAA-MNP (nanoscreenMAG-PAS, $200 \mathrm{~nm}$ ), and carboxymethyl-dextran-coated MNPs (fluidMAG-CMX; $200 \mathrm{~nm}$, denoted as CMXMNPs) were purchased from Chemicell (Berlin, Germany). Poly-L-lysine (PLL)-coated maghemite nanoparticles (PLLMNPs, $79 \mathrm{~nm}$ ) with positive and negative $\zeta$-potentials were prepared and characterized as described previously. ${ }^{31}$ Minimum essential media (MEM) and trypsin-EDTA were purchased from Gibco BRL (Grand Island, NY, USA). Penicillin/streptomycin/amphotericin B (PSA) was purchased from Upstate (Lake Placid, NY, USA). Fetal bovine serum (FBS), ammonium persulfate, potassium thiocyanate, cytochalasin D, and blebbistatin were purchased from Sigma-Aldrich (St. Louis, MO, USA). Wheat germ agglutinin (WGA), rhodamine-phalloidin, Alexa Fluor 568 goat anti-mouse secondary antibody, and 4',6-diamidino-2-phenylindole (DAPI) were purchased from Thermo Fisher Scientific (Waltham, MA, USA). Mouse anti-vinculin antibody was purchased from Santa Cruz Biotechnology, Inc. (Dallas, TX, USA). Six-well flexible-bottomed culture plates coated with collagen I (BioFlex ${ }^{\circledR}$ plates) were purchased from Flexcell ${ }^{\circledR}$ International Corporation (Burlington, NC, USA).

\section{Cell Culture}

Rat VSMCs with heterogeneous phenotype were isolated from spontaneously hypertensive rats as previously described, ${ }^{34}$ according to a protocol approved by Institutional Animal Care and Use Committee of Chang Gung University (CGU 108-041), which is certified by American Association for Accreditation of Laboratory Animal Care. The isolated VSMCs were cultured in MEM supplied with 10\% FBS and 1\% PSA mixture, maintained in a $37^{\circ} \mathrm{C}$ incubator supplied with $5 \%$ $\mathrm{CO}_{2}$, and used between passages 2 and 8. In some experiments, MNP uptake by LN-229 cells of a human glioma cell line (Bioresource Collection and Research Center, Food Industry Research and Development Institute, Taiwan) was determined, which were cultured in Dulbecco's modified minimal essential medium supplemented with $10 \%$ FBS and $1 \%$ PSA.

\section{Application of Mechanical Strain}

VSMCs were seeded and grown in collagen I-coated, flexible silicon membrane-bottomed 6-well culture plates until 80-90\% confluence. The culture plates were then placed on the Bioflex ${ }^{\circledR} 25 \mathrm{~mm}$ baseplate and connected to Flexcell ${ }^{\circledR}$ FX- $4000^{\mathrm{TM}}$ Tension System. The mechanical cyclic stretch was induced by vacuum to apply $1 \mathrm{~Hz}, 10 \%$ equibiaxial strain for various 
time periods. ${ }^{5}$ Three different strategies of mechanical stimulation were tested in this study, including (A) addition of MNPs after strain followed by 4-h incubation without strain; (B) addition of MNPs at the beginning of strain followed by 4-h incubation without strain; and (C) addition of MNPs after strain followed by additional 4-h strain. A home-made magnetic plate with 6 pieces of cylindrical $\mathrm{NdFeB}$ magnet ${ }^{35}$ was placed underneath the BioFlex ${ }^{\circledR}$ plates for 5 min after administration of MNPs $(100 \mu \mathrm{g} / \mathrm{mL})$ to ensure sedimentation, which can be a parameter that alters MNP internalization. ${ }^{32}$

\section{Determination of Cell-Associated MNPs (MNP cell $)$}

After incubation with MNPs, cells were gently washed twice with phosphate-buffered saline (PBS) and trypsinized for further analysis, as described previously. ${ }^{35}$ The cell pellet was treated with $10 \%$ hydrochloric acid at $55{ }^{\circ} \mathrm{C}$ for at least 4 $\mathrm{h}$, followed by addition of ammonium persulfate $(1 \mathrm{mg} / \mathrm{mL})$ and potassium thiocyanate $(1 \mathrm{M})$. Cell-associated iron was determined using VICTOR3 Multilabel Plate Reader (PerkinElmer, Waltham, MA, USA) at OD $_{490}$. A calibration curve was prepared under identical conditions with known amount of MNPs.

\section{Transmission Electron Microscopy (TEM)}

VSMCs in collagen I-coated BioFlex ${ }^{\circledR}$ plates were subjected to mechanical strain and MNPs, gently rinsed with PBS, and fixed with $4 \%$ paraformaldehyde. After fixation, cells were thoroughly rinsed with $\mathrm{ddH}_{2} \mathrm{O}$, followed by treatment with $1 \%$ osmium tetroxide with shaking at $5 \mathrm{rpm}$ for $1 \mathrm{~h}$. The samples were dehydrated with graded ethanol and embedded in Epon resin, with the silicon membrane carefully removed from the resin. Sections with $80 \mathrm{~nm}$ thickness were obtained, counterstained with $4 \%$ uranyl acetate in hydrogen peroxide for $2 \mathrm{~h}$ and $0.4 \%$ lead citrate for $10 \mathrm{~min}$, and examined with TEM (Jeol JEM-2000 EXII; JEOL USA, Inc., MA, USA). Image processing and analysis of intracellular vesicles were performed using Image $\mathrm{J}$ software.

\section{Scanning Electron Microscopy (SEM)}

VSMCs in collagen I-coated BioFlex ${ }^{\circledR}$ plates were subjected to mechanical strain and MNPs, rinsed with PBS, and fixed with $4 \%$ paraformaldehyde. After fixation, the membrane with the cells was carefully removed from the BioFlex ${ }^{\circledR}$ plates and placed in another culture plate, rinsed with $\mathrm{ddH}_{2} \mathrm{O}$, exposed to $1 \%$ osmium tetroxide with shaking at $5 \mathrm{rpm}$ for $1 \mathrm{~h}$. The samples were dehydrated with graded ethanol and then applied to critical point dryer for $2 \mathrm{~h}$. The samples were sputter-coated with 1-2 nm gold-palladium and examined with a field-emission scanning electron microscope (JEOL JSM-7500F, JEOL USA, Inc., MA, USA).

\section{Confocal Microscopy}

VSMCs were seeded and grown in BioFlex ${ }^{\circledR}$ plates until $80-90 \%$ confluence. In the fibronectin experiment, the cells were seeded and grown on fibronectin-coated coverslips. After applications of mechanical strain and treatments with green fluorescent MNPs, the cells were gently washed twice with PBS and fixed with 4\% paraformaldehyde. In the fibronectin experiment, the cells on fibronectin-coated coverslips were subjected to mouse anti-vinculin antibody and goat anti-mouse secondary antibody for the distribution of vinculins with WGA (a marker for membrane glycoproteins and the trans-Golgi in the cytoplasm) or rhodamine-phalloidin (a high-affinity F-actin probe) as morphologic indicators. All experiment samples were counterstained with DAPI and imaged with a Zeiss LSM 510 Meta laser confocal microscope (Carl Zeiss, Jena, Germany) equipped with 100×/1.4 oil immersion objective lens.

\section{Statistical Analysis}

Values are expressed as mean $\pm \mathrm{SE}$. Effects of treatment were examined by Student's $t$-test or 2-way analysis of variance (ANOVA) followed by Duncan's post hoc test when appropriate. Statistical significance was declared as $p<0.05$.

\section{Results}

Figure 1 illustrates effects of strain duration and MNP incubation time on MNP uptake by VSMCs. In response to various durations of cyclic strain followed by 4-h incubation with PAA-MNPs without strain, the cell-associated MNPs $\left(\mathrm{MNP}_{\text {cell }}\right)$ were reduced to $67 \%(16.9 \mathrm{pg} / \mathrm{cell})$ and $35 \%$ of control $(9.0 \mathrm{pg} / \mathrm{cell})$ after 4 and $12 \mathrm{~h}$ of cyclic strain, 

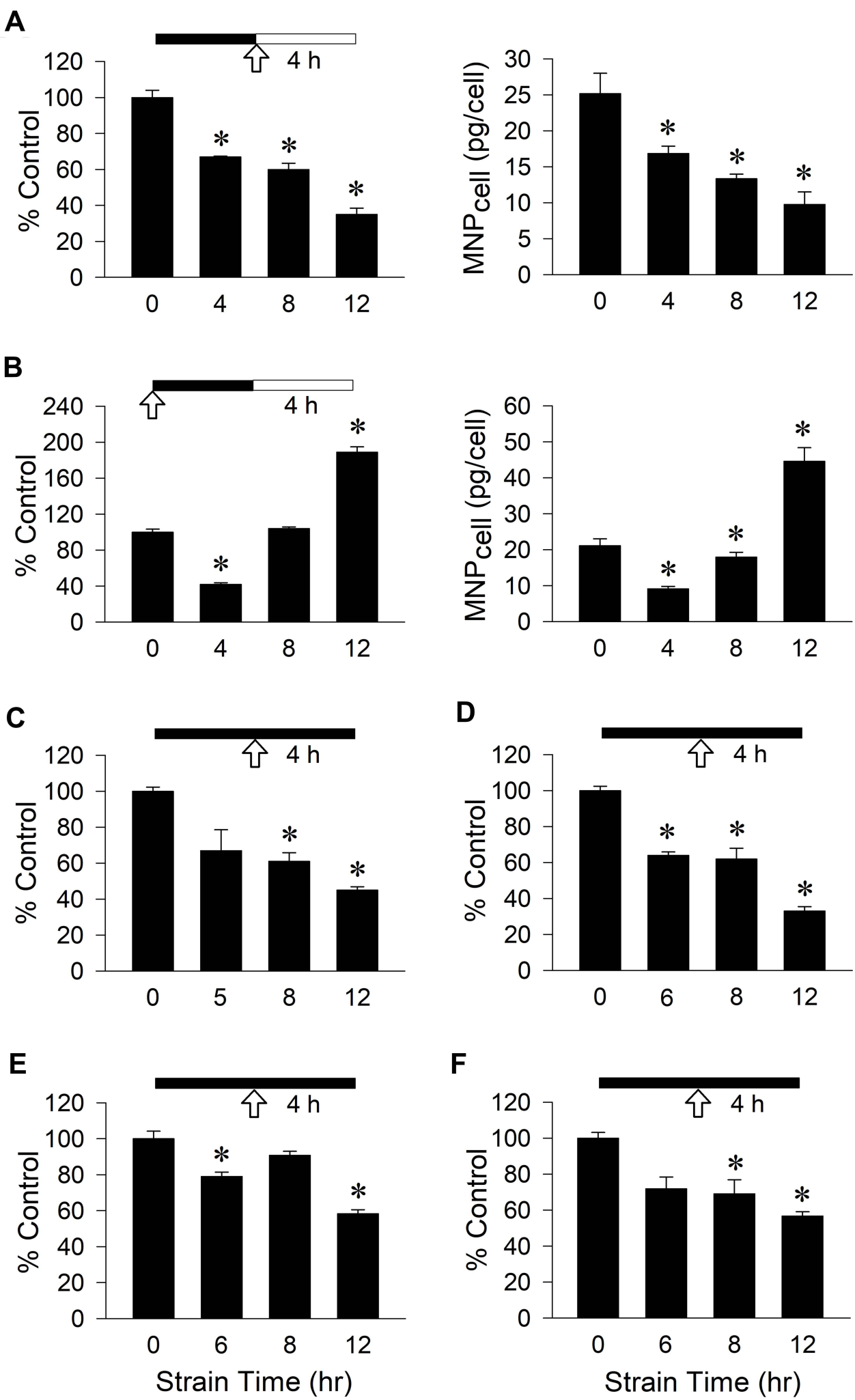

Figure I VSMC uptake of PAA-MNPs in response to mechanical strain with different strategies. Cyclic strain was applied as indicated by the solid bar for 4-12 $\mathrm{h}$ before or during incubation of $(\mathbf{A}-\mathbf{C})$ 200-nm or (D) 50-nm PAA-MNPs, or PLL-MNPs with (E) positive and (F) negative $\zeta$-potentials at $100 \mu g / \mathrm{mL}\left(25 \mu \mathrm{g} / \mathrm{cm}^{2}\right)$ with VSMCs. Cell-

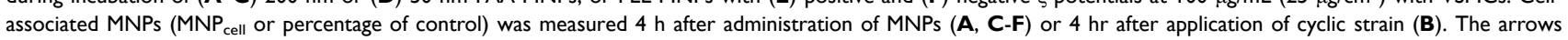
indicate the MNP administration; filled vs unfilled bars represent incubation with or without cyclic strain, respectively. Values are means \pm SEM ( $n=3$ ). $* p<0.05$ compared with corresponding values without strain. 
A

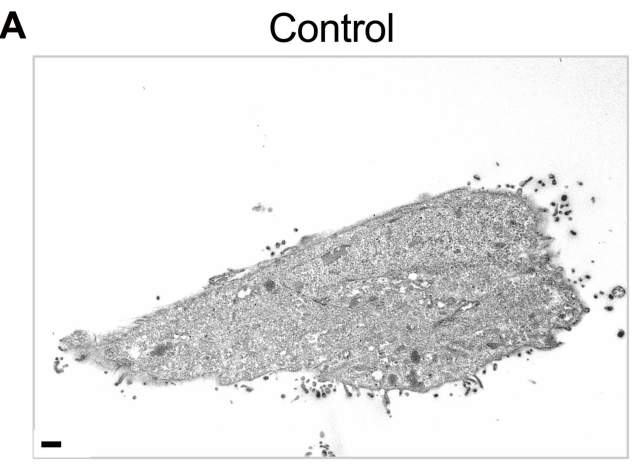

B

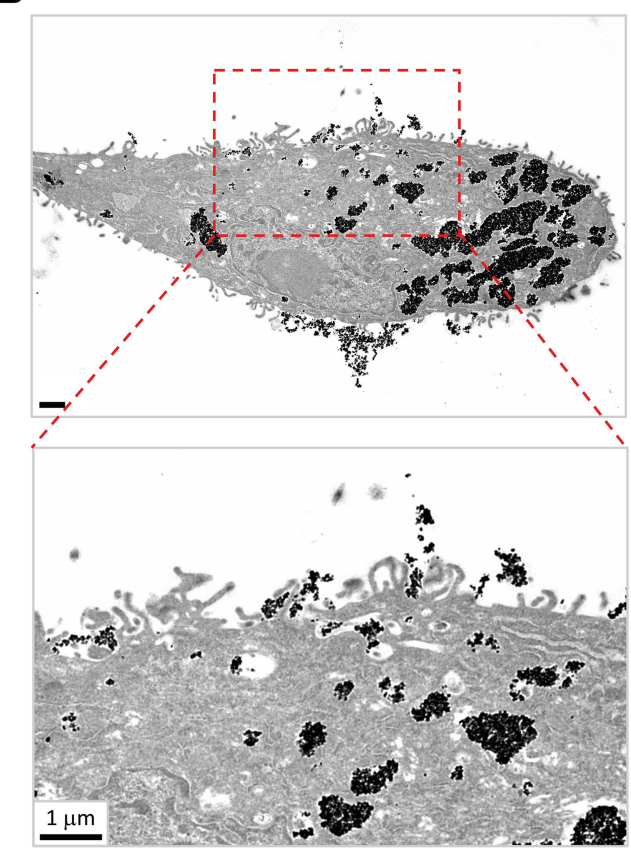

C

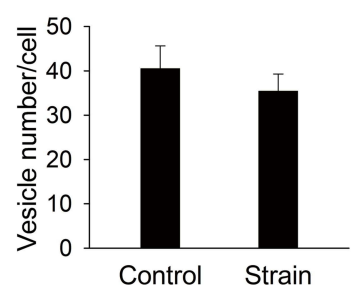

D

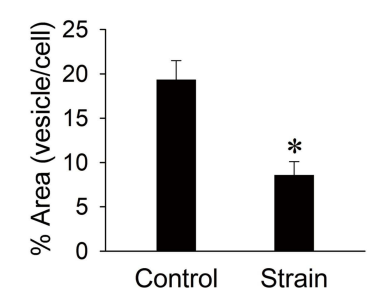

Strain
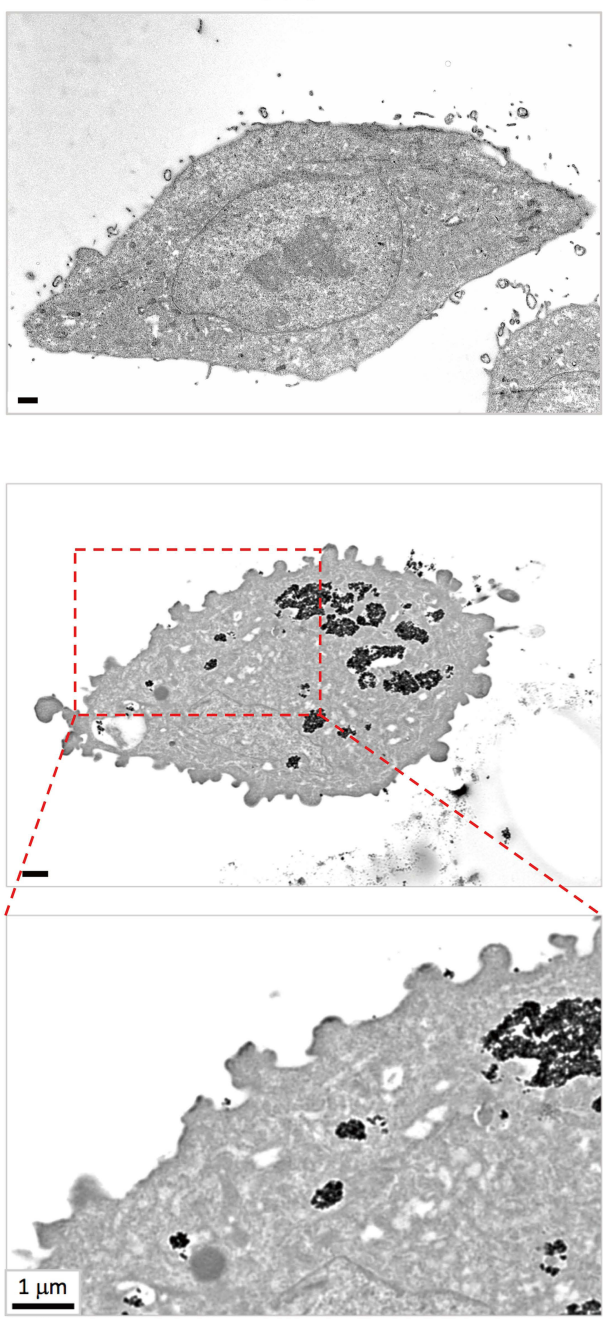

E

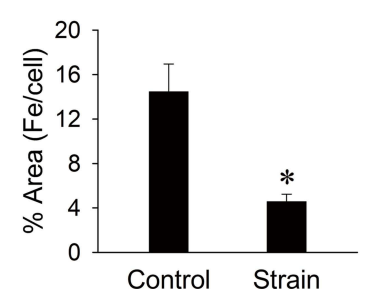

Figure 2 Changes in plasma membrane morphology were associated with reduced particle internalization in response to cyclic strain. VSMCs were subjected to cyclic strain for total of $12 \mathrm{~h}$ (A and B) with PAA-MNPs (100 $\left.\mathrm{\mu g} / \mathrm{mL} ; 25 \mu \mathrm{g} / \mathrm{cm}^{2}\right)$ added $8 \mathrm{~h}$ after initiation of strain (B and C). Cyclic strain-induced morphology changes from filopodia (B; left) to blunted protrusion (B; right) in the presence of PAA-MNPs are representative of 15 and 14 cells, respectively. The bottom panels (B) are amplifications of the areas in the rectangles. Quantitative analysis of intracellular (C) vesicle numbers, $(\mathbf{D})$ vesicle size, and $(\mathbf{E})$ areas of cells containing iron in TEM images of cells subjected to strain $(n=20)$ or without strain (control; $n=23$ ). Values are means \pm SEM. ${ }^{*} p<0.05$ compared with the corresponding control.

respectively (Figure 1A). When MNPs were added in the beginning of cyclic strain application (Figure 1B), cyclic strain reduced $\mathrm{MNP}_{\text {cell }}$ to $42 \%$ of control (8.4 pg/cell) after 4-h strain, but increased $\mathrm{MNP}_{\text {cell }}$ to 1.9 fold of control (38.3 pg/cell) after 12-h strain, probably due to an increment in overall incubation time with MNPs. Stimulation of physiological environment with continuous strain exerted a decrease pattern in $\mathrm{MNP}_{\text {cell }}$ over time. The $\mathrm{MNP}_{\text {cell }}$ was reduced to about $60 \%$ of control in both $200 \mathrm{~nm}$ (Figure 1C) vs $50 \mathrm{~nm}$ (Figure 1D) PAA-MNPs after 8-h strain, but further reduced to 45 vs $33 \%$ of control after 12 -h strain, respectively. In MNPs with different coating, size and $\zeta$-potential, cyclic strain also 


\section{A Control}
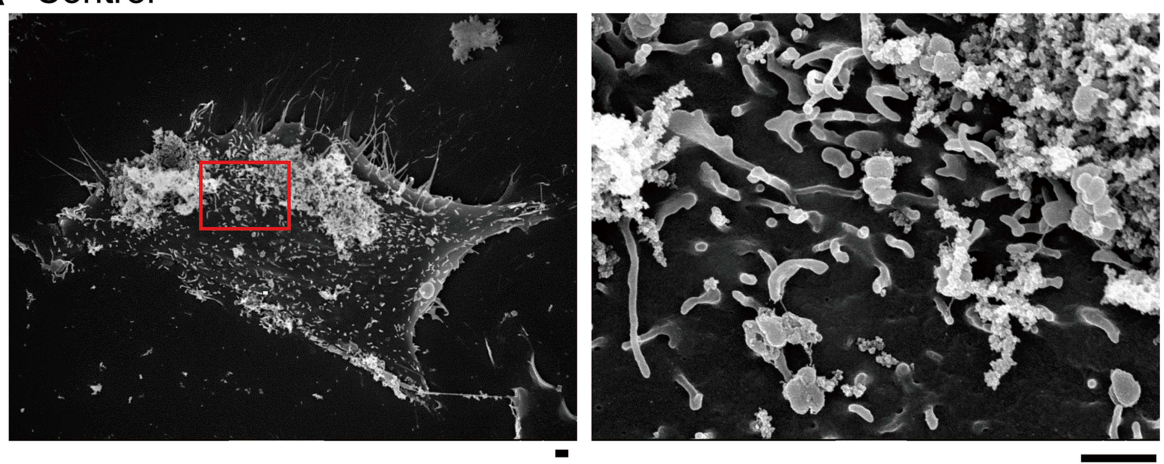

\section{B Cyclic Strain}
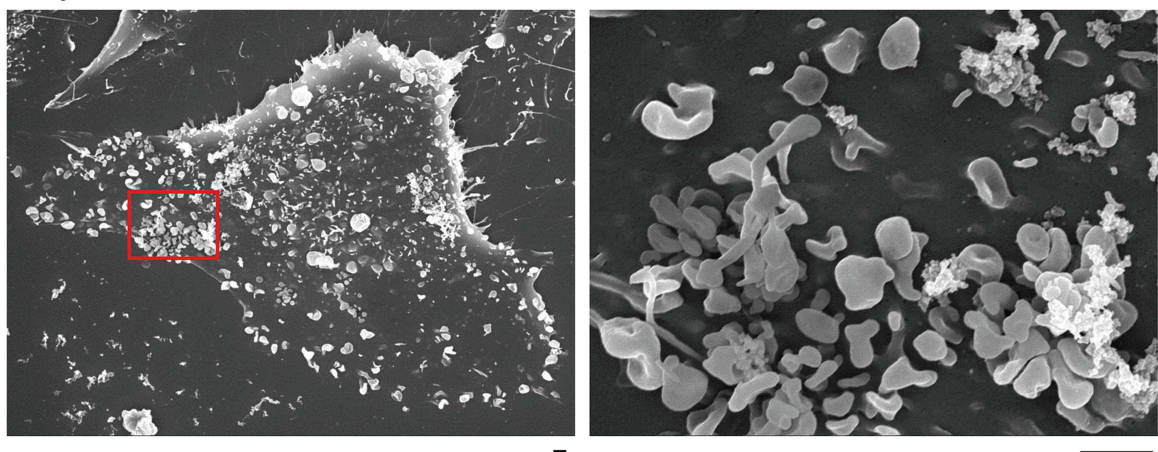

C

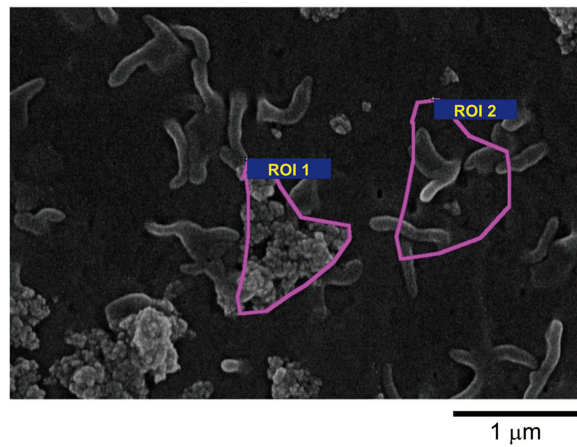

\begin{tabular}{ccccc}
\hline Element & \multicolumn{2}{c}{ ROI 1 } & \multicolumn{2}{c}{ ROI 2 } \\
\cline { 2 - 4 } & $\begin{array}{c}\text { Weight } \\
(\%)\end{array}$ & $\begin{array}{c}\text { Atomic } \\
(\%)\end{array}$ & $\begin{array}{c}\text { Weight } \\
(\%)\end{array}$ & $\begin{array}{c}\text { Atomic } \\
(\%)\end{array}$ \\
\hline C & 59.0 & 75.9 & 53.8 & 64.4 \\
O & 18.6 & 17.9 & 36.9 & 33.2 \\
Fe & $\mathbf{2 2 . 4}$ & $\mathbf{6 . 2}$ & $\mathbf{9 . 3}$ & $\mathbf{2 . 4}$ \\
\hline
\end{tabular}

Figure 3 Representative SEM images of VSMCs subjected to mechanical strain during incubation with PAA-MNPs. (A) VSMCs were subjected to cyclic strain for 12 h or in steady culture conditions (control). (B) Cyclic strain was applied for $8 \mathrm{~h}$ before administration of PAA-MNPs $\left(25 \mu \mathrm{g} / \mathrm{mL} ; 6.25 \mu \mathrm{g} / \mathrm{cm}^{2}\right)$, followed by additional $4 \mathrm{~h}$ of strain. The right panels are amplification of the areas as indicated on the left in (A and B). Scale bars indicate I $\mu \mathrm{m}$. (C) SEM/EDS surface elemental analysis was conducted in the indicated areas (ROI I \& 2) of the surface of VSMCs exposed to PAA-MNPs in static conditions (Bold values).

reduced $\mathrm{MNP}_{\text {cell }}$ in a similar manner. In PLL-MNPs with positive $\zeta$-potential, the $\mathrm{MNP}_{\text {cell }}$ was reduced by $21 \%$ after 6-h strain (Figure 1E), whereas $\mathrm{MNP}_{\text {cell }}$ of PLL-MNPs with negative $\zeta$-potential reduced $31 \%$ after 8 -h strain (Figure $1 \mathrm{~F}$ ). The $\mathrm{MNP}_{\text {cell }}$ was further reduced to 58 or $57 \%$ of control after 12-h strain in the groups of PLL-MNPs with positive or negative $\zeta$-potential, respectively $(\mathrm{n}=4)$.

Effect of cyclic strain on PAA-MNP uptake and cell morphologic change was observed by TEM. Figure 2 illustrates representative results in VSMCs subjected to cyclic strain for $12 \mathrm{~h}$. When VSMCs were not in contact with the particles, plasma membrane remained relatively smooth (Figure 2A). PAA-MNPs stimulated formation of thin protrusions, also known as filopodia, on the cell surface, which transformed to short cylindrical-like structures after $12 \mathrm{~h}$ of cyclic strain (Figure 2B). It was noted that dense structure appeared at the tip of some protrusions. Despite unaltered number of the vesicles/cell (Figure 2C), the area of vesicle per cell (Figure 2D) and the iron-containing area/cell (Figure 2E) was reduced by $56 \%$ and $68 \%$ after cyclic strain, respectively. 
A
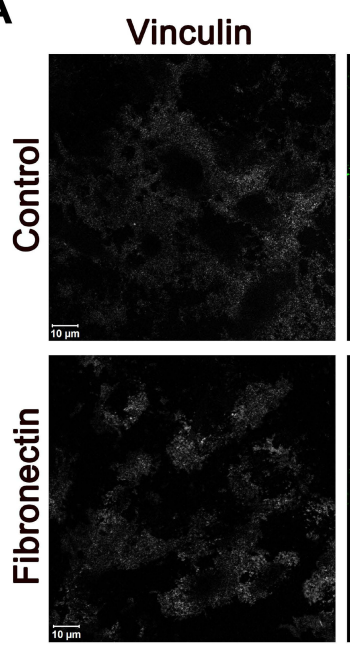

B
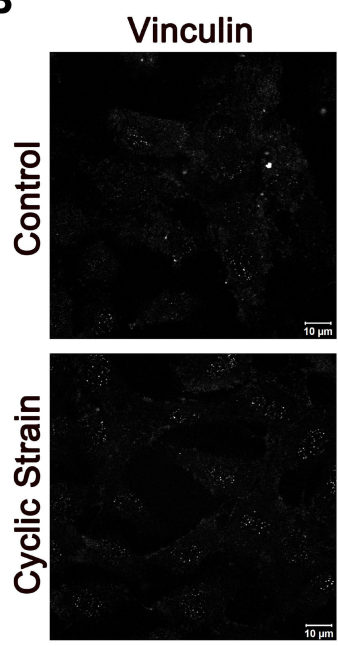

MNP
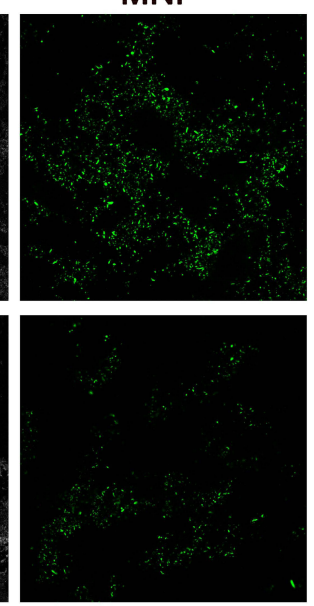

MNP
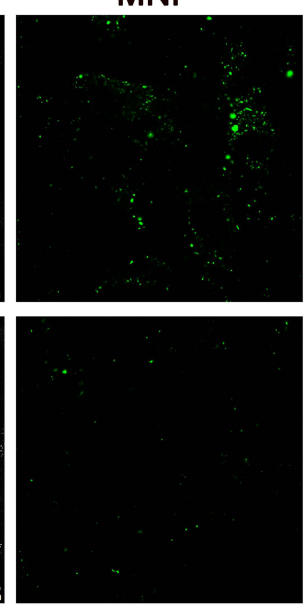

WGA
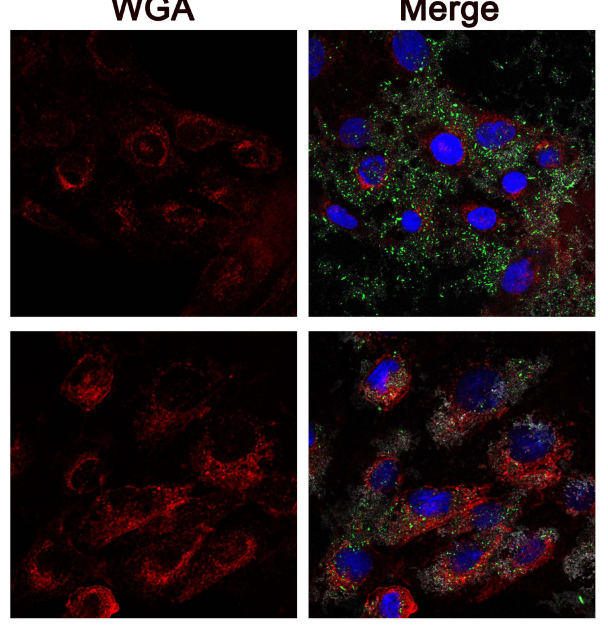

WGA
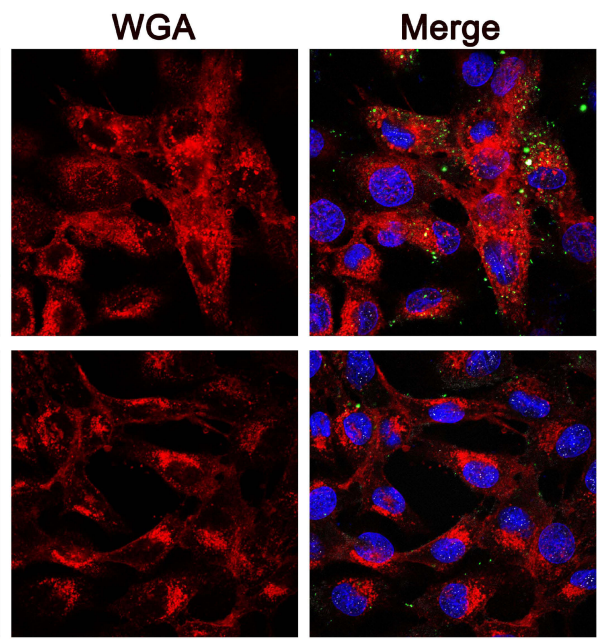

Figure 4 Representative confocal images of PAA-MNPs uptake by VSMCs subjected to cyclic strain. (A) VSMCs were incubated with PAA-MNPs (I00 $\mu$ g/mL; $25 \mu g / \mathrm{cm}^{2}$, green) in culture dishes coated with fibronectin for $4 \mathrm{~h}$; (B) VSMCs were subjected to cyclic strain for $8 \mathrm{~h}$ before administration of PAA-MNPs, followed by additional $4 \mathrm{~h}$ of cyclic strain with PAA-MNPs in the culture media. Cell membrane, nuclei and focal adhesions were stained by WGA (red), DAPI (blue), and mouse anti-vinculin antibody (white), respectively.

Cyclic strain-induced 3-D morphological change on the cell membrane reveals a similar pattern, as observed by SEM. There were visible filopodia structures in the static state (Figure 3A) as well as after cyclic strain treatment (Figure 3B). The shape of the filopodia observed by SEM was slender/spaghetti-like vs stubby/mushroom-like in the control vs cyclic strain group, respectively. In addition, the elemental analysis indicated the presence of a significantly higher amount of iron in the wadding structures ROI 1 than in the filopodia ROI 2 (Figure 3C), suggesting that these wadding structures are the iron oxide-containing particles on the surface of the cells.

It has been well-accepted that mechanical strain alters cytoskeleton through controlling of FA dynamics. To determine whether FA aggregation may be associated with particle internalization, MNPs were incubated with VSMCs cultured on the surface with fibronectin coating to induce FA assembly without application of cyclic strain. Figure 4A illustrates that fibronectin induced vinculin aggregation, which was associated with much less uptake of MNPs. It was noted that the WGA signal distributed at peri-nucleus space regardless of fibronectin coating. Figure 4B illustrates that cyclic strain also reduced $\mathrm{MNP}_{\text {cell, }}$, as in Figure 1, with condensed WGA signal to the peri-nucleus space. In addition, co-localization of MNPs and vinculins was noted in all groups studied, suggesting that particle internalization may be associated with a vinculin-dependent mechanism. 

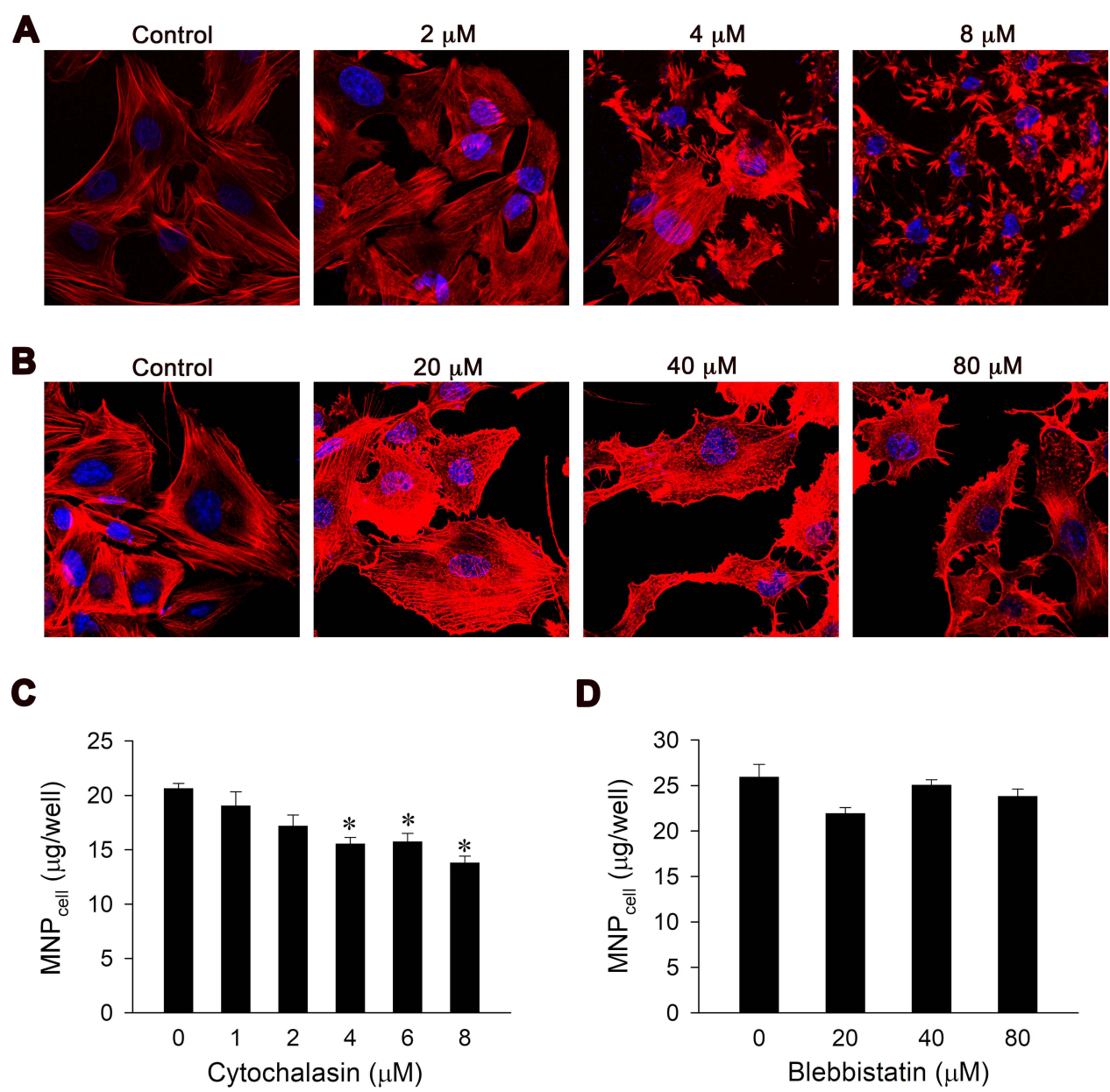

D

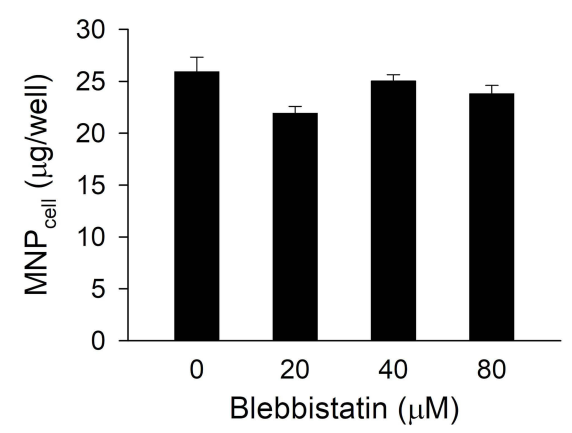

Figure 5 Cytochalasin D, but not blebbistatin, reduced VSMC uptake of PAA-MNPs in a concentration-dependent manner. Confocal images of VSMCs with I-h pretreatment of $(\mathbf{A})$ cytochalasin D or (B) blebbistatin at different concentrations. The cytoskeleton and nuclei were stained by phalloidin (red) and DAPI (blue), respectively. VSMCs were pretreated with cytochalasin D (C) or blebbistatin (D) for I h followed by incubation with PAA-MNPs (I00 $\mu \mathrm{g} / \mathrm{mL} ; 25 \mu g / \mathrm{cm}^{2}$ ) for $3 \mathrm{~h}$ prior to measurement. Values are means $\pm \operatorname{SEM}(n=4)$. ${ }_{p} p<0.05$ compared with the control value.

Direct regulations of cytoskeleton were then evaluated using cytochalasin D and blebbistatin, inhibitors of actin polymerization and myosin II ATPase, ${ }^{36,37}$ respectively. Confocal microscopic images demonstrated that both cytochalasin D and blebbistatin disrupted cytoskeleton architecture with increase in concentrations (Figure 5A and B). However, cytochalasin $\mathrm{D}$, but not blebbistatin, significantly reduced MNP uptake in a concentration-dependent manner. $\mathrm{MNP}_{\text {cell }}$ reduced to $83-67 \%$ of control by $2-8 \mu \mathrm{M}$ of cytochalasin D (Figure $5 \mathrm{C}$ ), whereas no significant difference in $\mathrm{MNP}_{\text {cell }}$ was observed after blebbistatin treatment (Figure 5D). These results suggest that the internalization of MNPs is directly regulated by actin dynamics.

In addition to VSMCs, cyclic strain attenuated MNP uptake by LN-229 cells in a time-dependent manner (Figure 6). Application of cyclic strain for $12 \mathrm{~h}$ followed by 4 -h incubation without strain reduced $\mathrm{MNP}_{\text {cell }}$ to $87 \%$ of control; application of cyclic strain for $12 \mathrm{~h}$ followed by additional 4-h strain reduced $\mathrm{MNP}_{\text {cell }}$ to $71 \%$ of control.

\section{Discussion}

To the best of our knowledge, this study is the first demonstration of cyclic strain-sensitive internalization of nanoparticles with a variety of coatings by VSMCs, suggesting an active involvement of the mechanical force-regulated signaling in the nanoparticle uptake mechanism. Although the effects are profound and not reversible after $4 \mathrm{~h}$, an increased incubation time reversed the effect of cyclic strain on MNP uptake; the effects of cyclic strain are unlikely to be due to 

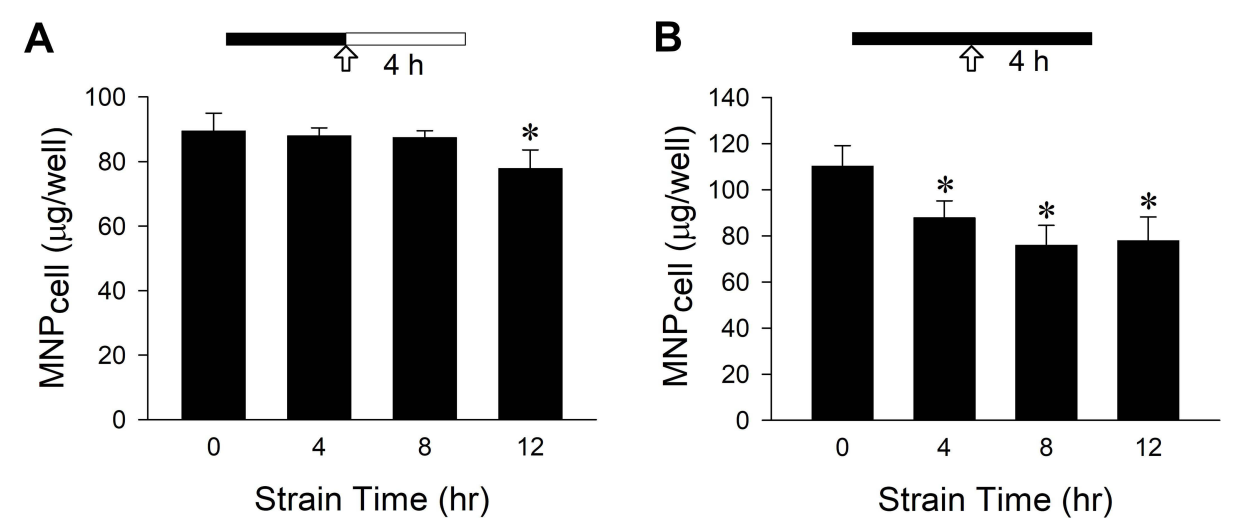

Figure 6 Mechanical strain attenuated PAA-MNPs uptake by LN-229 cells with time. Mechanical strain was applied as indicated by the solid bar for 4-12 h (A) before or (B) during incubation of LN-229 cells with PAA-MNPs $\left(100 \mu \mathrm{g} / \mathrm{mL} ; 25 \mu \mathrm{g} / \mathrm{cm}^{2}\right)$. The arrows indicate the MNP administration; filled vs unfilled bars represent incubation with or without cyclic strain, respectively. Values are means \pm SEM $(n=3) . * p<0.05$ compared with corresponding values without strain.

cytotoxicity or reduction in cell numbers. Physiological cyclic strain upon vascular cells, and tumor cells to a lesser degree, may hinder nanoparticle-cell interaction. Therefore, study of cells in physiological conditions with mechanical dynamics can be crucial in theranostic applications of nanoparticles, especially in the circulatory system.

In spite of many different endocytosis pathways that mediate cellular uptake of nanoparticles, ${ }^{14}$ TEM studies illustrated plasma membrane protrusions with large vesicles in the presence of MNPs (Figure 2B), suggesting uptake via macropinocytosis in VSMCs. Although MNPs in size ranging from 69-200 nm tested in this study are relatively small, they are likely to aggregate in magnetic field applied briefly to facilitate sedimentation. Therefore, macropinocytosis for uptake of large particle aggregates is probably the major mechanism of internalization, which is characterized by formation of filopodia with cytoskeleton to support membrane protrusion. ${ }^{14}$ In addition, the presence of plasma proteins and formation of protein corona on the surface of MNPs may further augment aggregation and increase the size of the aggregate. ${ }^{38,39}$ In the presence of plasma proteins as in the culture medium, positive or negative $\zeta$-potentials of nanoparticles tend to become sheltered, which may contribute to further increase in the size of the aggregates. ${ }^{40}$ Our results are consistent with previous findings that PLL-MNPs with positive or negative $\zeta$-potentials exhibited similar pattern of interaction with glioma cells in culture. ${ }^{31}$

Intriguingly, morphology of MNP-induced filopodia became much blunted after application of cyclic strain (Figure 2B). The results are in parallel with SEM observation of stubby/mushroom-like protrusions (Figure 3B), suggesting that cyclic strain may increase the membrane tension. These morphological changes are consistent with previous findings that the lamellipodial extension rate correlates inversely with membrane tension. ${ }^{41,42}$ Although the number of inward vesicles was not significantly reduced in control vs strained cells, the percentage of vesicle area decreased significantly, suggesting the regulation of inward vesicles may be affected in VSMCs subjected to cyclic strain.

Several studies have demonstrated inconsistent response of nanoparticle internalization to mechanical strain; ${ }^{33,43,44}$ the discrepancy of the strain effect on nanoparticle internalization can be attributed to the size/coating of nanoparticles and cell types. However, when VSMCs were under strain in the current study, significant reduction in nanoparticle uptake was observed in spite of different properties of nanoparticles used in the current study. The results are in parallel with the study of Freese et al. ${ }^{2}$ demonstrating that cyclic strain reduced nanoparticle uptake by endothelial cells, which was not due to induction of stress, inflammation or enhanced exocytosis, but rather a result of decreased endocytosis. The disturbances of cellular signaling, such as actin dynamics, by external stimuli may be the mechanism underneath the jeopardized internalization, rather than interaction occurring at the nano-bio interface.

Since endocytosis may occur at filopodia, ${ }^{23,45,46}$ condensed protrusions observed on the surface of VSMCs after cyclic strain are consistent with the previous findings that reduction in filopodia was observed after inhibition of endocytosis. ${ }^{45,47}$ It is likely that cyclic strain-induced interruption of actin assembly and thus filopodia formation may jeopardize MNP internalization by VSMCs. The finding is consistent with previous studies demonstrating that 
cytochalasin $\mathrm{D},{ }^{48}$ but not blebbistatin, ${ }^{49}$ reduced filopodia formation, suggesting an important role of filopodia in nanoparticle internalization by VSMCs. Alternatively, the strain also increases plasma membrane apparent tension, ${ }^{50,51}$ which reduces membrane protrusion/invaginations or blebs passively, as observed in the current study. The reduced $\mathrm{MNP}_{\text {cell }}$ is also consistent with previous findings that the increased membrane tension exhibits an inverse relation with the endocytosis rate. ${ }^{20}$ Nevertheless, fibronectin-induced FA formation and trans-Golgi condensation, as demonstrated with vinculin distribution and WGA staining, respectively, were also accompanied with reduction in MNP uptake (Figure 4A), suggesting an altered actin assembly may be the major mechanism underlying the effects of cyclic strain on MNP uptake. Since cytochalasin D reduced MNP internalization in a concentration-dependent manner, polymerization of actin in the cytoskeleton may be critical in the process of MNP uptake by VSMCs. Alternatively, cyclic straininduced reduction in $\mathrm{MNP}_{\text {cell }}$ may be mediated by an increase in exocytosis; however, previous study in endothelial cell does not support the involvement of exocytosis. ${ }^{2}$

Reduced MNP uptake in response to cyclic strain was also demonstrated with confocal microscopy, in addition to fibronectin-coating. VSMCs cultured on fibronectin-coated surface recruit more vinculin clustering in FA (Figure 4A), which was consistent with the previous finding demonstrating protein clusters in FA in the cells cultured on the fibronectin-coated surface. ${ }^{52}$ Since WGA conjugates are commonly used as markers for glycoproteins on the plasma membrane and the trans-Golgi in the cytoplasm, ${ }^{53}$ the peri-nucleus condensation of WGA signal (Figure 4B) is consistent with the previous finding of trans-Golgi clustering in response to cyclic strain. ${ }^{54}$

In summary, this study has demonstrated that cyclic strain affects the interaction of MNPs and VSMCs in culture, which helps to solidify the importance of the establishment of physiologically relevant in vitro models for study of nanoparticle behaviors in the nano-bio interface. Since VSMCs are subject to a very wide range of cyclic strain that is transduced from the tissue to the cell level in vivo, understanding the effects of such bio-mechanical factors helps to dictate the process and phenomena of physiological activities. Therefore, interaction between engineered nanoparticles and the biological system may affect how these nanoparticles can be employed in theranostic applications.

\section{Conclusion}

Cyclic strain changed the morphology of filopodia triggered by MNPs and reduced internalized MNPs in VSMCs. Our findings reveal potential challenges for intracellular delivery of nanocarriers to organ/tissue subjected to high mechanical stress, such as VSMCs in the vascular wall. Nevertheless, the effects of cyclic strain on the vascular wall may not affect application of nanocomposites for the targets in blood circulation, such as the thrombus or emboli, in treatment of thromboembolic diseases. Our finding may provide an inspiration for future development of nanoparticles and prediction of nanoparticle biodistribution.

\section{Acknowledgments}

The authors thank Sonya Y. Hsueh for assistance with the graphic abstract, and the Microscopy Center at Chang Gung University for technical assistance. This work was supported by Ministry of Science and Technology of the Republic of China (MOST 107-2311-B-182-002-; MOST 109-2923-B-182-001-MY3), Chang Gung Memorial Hospital (BMRP432), and the Czech Science Foundation (No. 20-02177J).

\section{Disclosure}

The authors report no conflicts of interest in this work.

\section{References}

1. Panyam J, Labhasetwar V. Dynamics of endocytosis and exocytosis of poly(D,L-lactide-co-glycolide) nanoparticles in vascular smooth muscle cells. Pharm Res. 2003;20(2):212-220. doi:10.1023/A:1022219003551

2. Freese C, Schreiner D, Anspach L, et al. In vitro investigation of silica nanoparticle uptake into human endothelial cells under physiological cyclic stretch. Part Fibre Toxicol. 2014;11:68. doi:10.1186/s12989-014-0068-y

3. Lu YC, Luo PC, Huang CW, et al. Augmented cellular uptake of nanoparticles using tea catechins: effect of surface modification on nanoparticle-cell interaction. Nanoscale. 2014;6(17):10297-10306. doi:10.1039/C4NR00617H

4. Kakisis JD, Liapis CD, Sumpio BE. Effects of cyclic strain on vascular cells. Endothelium. 2004;11(1):17-28. doi:10.1080/10623320490432452 
5. Su BY, Shontz KM, Flavahan NA, Nowicki PT. The effect of phenotype on mechanical stretch-induced vascular smooth muscle cell apoptosis. J Vasc Res. 2006;43(3):229-237. doi:10.1159/000091102

6. Ando J, Yamamoto K. Effects of shear stress and stretch on endothelial function. Antioxid Redox Signal. 2011;15(5):1389-1403. doi:10.1089/ ars.2010.3361

7. Fang Y, Wu D, Birukov KG. Mechanosensing and mechanoregulation of endothelial cell functions. Compr Physiol. 2019;9(2):873-904.

8. van Engeland NCA, Pollet A, den Toonder JMJ, Bouten CVC, Stassen O, Sahlgren CM. A biomimetic microfluidic model to study signalling between endothelial and vascular smooth muscle cells under hemodynamic conditions. Lab Chip. 2018;18(11):1607-1620. doi:10.1039/ C8LC00286J

9. Jufri NF, Mohamedali A, Avolio A, Baker MS. Mechanical stretch: physiological and pathological implications for human vascular endothelial cells. Vasc Cell. 2015;7:8. doi:10.1186/s13221-015-0033-z

10. Lim CG, Jang J, Kim C. Cellular machinery for sensing mechanical force. BMB Rep. 2018;51(12):623-629. doi:10.5483/BMBRep.2018.51.12.237

11. Rensen SS, Doevendans PA, van Eys GJ. Regulation and characteristics of vascular smooth muscle cell phenotypic diversity. Neth Heart J. 2007;15 (3):100-108. doi:10.1007/BF03085963

12. Chen J, Zhou Y, Liu S, Li C. Biomechanical signal communication in vascular smooth muscle cells. J Cell Commun Signal. 2020;14(4):357-376. doi:10.1007/s12079-020-00576-1

13. Hu X, Zhao P, Lu Y, Liu Y. ROS-based nanoparticles for atherosclerosis treatment. Materials (Basel). 2021;14(22):6921. doi:10.3390/ma14226921

14. Behzadi S, Serpooshan V, Tao W, et al. Cellular uptake of nanoparticles: journey inside the cell. Chem Soc Rev. 2017;46(14):4218-4244. doi:10.1039/C6CS00636A

15. Oh N, Park JH. Endocytosis and exocytosis of nanoparticles in mammalian cells. Int J Nanomedicine. 2014;9(Suppl1):51-63.

16. Zhang S, Gao H, Bao G. Physical principles of nanoparticle cellular endocytosis. ACS Nano. 2015;9(9):8655-8671. doi:10.1021/acsnano.5b03184

17. Yameen B, Choi WI, Vilos C, Swami A, Shi J, Farokhzad OC. Insight into nanoparticle cellular uptake and intracellular targeting. $J$ Control Release. 2014;190:485-499. doi:10.1016/j.jconrel.2014.06.038

18. Swanson JA. Shaping cups into phagosomes and macropinosomes. Nat Rev Mol Cell Biol. 2008;9(8):639-649. doi:10.1038/nrm2447

19. Mooren OL, Galletta BJ, Cooper JA. Roles for actin assembly in endocytosis. Annu Rev Biochem. 2012;81:661-686. doi:10.1146/annurevbiochem-060910-094416

20. Gauthier NC, Masters TA, Sheetz MP. Mechanical feedback between membrane tension and dynamics. Trends Cell Biol. 2012;22(10):527-535. doi:10.1016/j.tcb.2012.07.005

21. Carlsson AE. Membrane bending by actin polymerization. Curr Opin Cell Biol. 2018;50:1-7. doi:10.1016/j.ceb.2017.11.007

22. Blanchoin L, Boujemaa-Paterski R, Sykes C, Plastino J. Actin dynamics, architecture, and mechanics in cell motility. Physiol Rev. 2014;94 (1):235-263. doi:10.1152/physrev.00018.2013

23. Gallop JL. Filopodia and their links with membrane traffic and cell adhesion. Semin Cell Dev Biol. 2020;102:81-89. doi:10.1016/j. semcdb.2019.11.017

24. Mogilner A. On the edge: modeling protrusion. Curr Opin Cell Biol. 2006;18(1):32-39. doi:10.1016/j.ceb.2005.11.001

25. Ohashi K, Fujiwara S, Mizuno K. Roles of the cytoskeleton, cell adhesion and rho signalling in mechanosensing and mechanotransduction. J Biochem. 2017;161(3):245-254.

26. Bauer MS, Baumann F, Daday C, et al. Structural and mechanistic insights into mechanoactivation of focal adhesion kinase. Proc Natl Acad Sci U S A. 2019;116(14):6766-6774. doi:10.1073/pnas.1820567116

27. Wrighton KH. Cell adhesion: the 'ins' and 'outs' of integrin signalling. Nat Rev Mol Cell Biol. 2013;14(12):752.

28. Xiao Y, Du J. Superparamagnetic nanoparticles for biomedical applications. J Mater Chem B. 2020;8(3):354-367. doi:10.1039/C9TB01955C

29. Revia RA, Zhang M. Magnetite nanoparticles for cancer diagnosis, treatment, and treatment monitoring: recent advances. Mater Today (Kidlington). 2016;19(3):157-168. doi:10.1016/j.mattod.2015.08.022

30. Ulbrich K, Holá K, Šubr V, Bakandritsos A, Tuček J, Zbořil R. Targeted drug delivery with polymers and magnetic nanoparticles: covalent and noncovalent approaches, release control, and clinical studies. Chem Rev. 2016;116(9):5338-5431.

31. Siow WX, Chang YT, Babič M, Lu YC, Horák D, Ma YH. Interaction of poly-1-lysine coating and heparan sulfate proteoglycan on magnetic nanoparticle uptake by tumor cells. Int J Nanomedicine. 2018;13:1693-1706. doi:10.2147/IJN.S156029

32. Dabaghi M, Hilger I. Magnetic nanoparticles behavior in biological solutions; the impact of clustering tendency on sedimentation velocity and cell uptake. Materials (Basel). 2020;13(7):1644. doi:10.3390/ma13071644

33. Rouse JG, Haslauer CM, Loboa EG, Monteiro-Riviere NA. Cyclic tensile strain increases interactions between human epidermal keratinocytes and quantum dot nanoparticles. Toxicol in Vitro. 2008;22(2):491-497. doi:10.1016/j.tiv.2007.10.010

34. Ma YH, Wei HW, Su KH, Ives HE, Morris RC. Chloride-dependent calcium transients induced by angiotensin II in vascular smooth muscle cells. Am J Physiol Cell Physiol. 2004;286(1):C112-118. doi:10.1152/ajpcell.00605.2002

35. Lu YC, Chang FY, Tu SJ, Chen JP, Ma YH. Cellular uptake of magnetite nanoparticles enhanced by NdFeB magnets in staggered arrangement. J Magn Magn Mater. 2017;427:71-80. doi:10.1016/j.jmmm.2016.11.010

36. Casella JF, Flanagan MD, Lin S. Cytochalasin D inhibits actin polymerization and induces depolymerization of actin filaments formed during platelet shape change. Nature. 1981;293(5830):302-305. doi:10.1038/293302a0

37. Kovács M, Tóth J, Hetényi C, Málnási-Csizmadia A, Sellers JR. Mechanism of blebbistatin inhibition of myosin II. J Biol Chem. 2004;279 (34):35557-35563. doi:10.1074/jbc.M405319200

38. Sakulkhu U, Mahmoudi M, Maurizi L, Salaklang J, Hofmann H. Protein Corona composition of superparamagnetic iron oxide nanoparticles with various physico-chemical properties and coatings. Sci Rep. 2014;4:5020. doi:10.1038/srep05020

39. Kokkinopoulou M, Simon J, Landfester K, Mailänder V, Lieberwirth I. Visualization of the protein Corona: towards a biomolecular understanding of nanoparticle-cell-interactions. Nanoscale. 2017;9(25):8858-8870. doi:10.1039/C7NR02977B

40. Chiu CY, Chung TW, Chen SY, Ma YH. Effects of PEGylation on capture of dextran-coated magnetic nanoparticles in microcirculation. Int J Nanomedicine. 2019;14:4767-4780.

41. Raucher D, Sheetz MP. Cell spreading and lamellipodial extension rate is regulated by membrane tension. J Cell Biol. 2000;148(1):127-136. doi: $10.1083 /$ jcb.148.1.127 
42. Batchelder EL, Hollopeter G, Campillo C, et al. Membrane tension regulates motility by controlling lamellipodium organization. Proc Natl Acad Sci U S A. 2011;108(28):11429-11434. doi:10.1073/pnas.1010481108

43. Hu J, Liu Y. Cyclic strain enhances cellular uptake of nanoparticles. J Nanomater. 2015;2015:1-8.

44. Freese C, Anspach L, Deller RC, et al. Gold nanoparticle interactions with endothelial cells cultured under physiological conditions. Biomater Sci. 2017;5(4):707-717. doi:10.1039/C6BM00853D

45. Nozumi M, Nakatsu F, Katoh K, Igarashi M. Coordinated movement of vesicles and actin bundles during nerve growth revealed by superresolution microscopy. Cell Rep. 2017;18(9):2203-2216. doi:10.1016/j.celrep.2017.02.008

46. Onishi K, Shafer B, Lo C, Tissir F, Goffinet AM, Zou Y. Antagonistic functions of Dishevelleds regulate Frizzled3 endocytosis via filopodia tips in Wnt-mediated growth cone guidance. J Neurosci. 2013;33(49):19071-19085. doi:10.1523/JNEUROSCI.2800-13.2013

47. Bu W, Chou AM, Lim KB, Sudhaharan T, Ahmed S. The Toca-1-N-WASP complex links filopodial formation to endocytosis. J Biol Chem. 2009;284(17):11622-11636. doi:10.1074/jbc.M805940200

48. Dent EW, Kwiatkowski AV, Mebane LM, et al. Filopodia are required for cortical neurite initiation. Nat Cell Biol. $2007 ; 9(12): 1347-1359$. doi:10.1038/ncb1654

49. Young LE, Heimsath EG, Higgs HN. Cell type-dependent mechanisms for formin-mediated assembly of filopodia. Mol Biol Cell. 2015;26 (25):4646-4659. doi:10.1091/mbc.E15-09-0626

50. Le Roux AL, Quiroga X, Walani N, Arroyo M, Roca-Cusachs P. The plasma membrane as a mechanochemical transducer. Philos Trans R Soc Lond B Biol Sci. 2019;374(1779):20180221. doi:10.1098/rstb.2018.0221

51. Qi YX, Han Y, Jiang ZL. Mechanobiology and vascular remodeling: from membrane to nucleus. Adv Exp Med Biol. $2018 ; 1097: 69-82$.

52. Li W, Sancho A, Chung WL, et al. Differential cellular responses to adhesive interactions with galectin-8- and fibronectin-coated substrates. $J$ Cell Sci. 2021;134(8):jes252221. doi:10.1242/jcs.252221

53. Chazotte B. Labeling membrane glycoproteins or glycolipids with fluorescent wheat germ agglutinin. Cold Spring Harb Protoc. 2011;5:pdb. prot5623. doi:10.1101/pdb.prot5623

54. Dhein S, Schreiber A, Steinbach S, et al. Mechanical control of cell biology. Effects of cyclic mechanical stretch on cardiomyocyte cellular organization. Prog Biophys Mol Biol. 2014;115(2-3):93-102. doi:10.1016/j.pbiomolbio.2014.06.006

International Journal of Nanomedicine

Dovepress

\section{Publish your work in this journal}

The International Journal of Nanomedicine is an international, peer-reviewed journal focusing on the application of nanotechnology in diagnostics, therapeutics, and drug delivery systems throughout the biomedical field. This journal is indexed on PubMed Central, MedLine, CAS, SciSearch ${ }^{\mathbb{R}}$, Current Contents ${ }^{\mathbb{B}} /$ Clinical Medicine, Journal Citation Reports/Science Edition, EMBase, Scopus and the Elsevier Bibliographic databases. The manuscript management system is completely online and includes a very quick and fair peer-review system, which is all easy to use. Visit http:// www.dovepress.com/testimonials.php to read real quotes from published authors.

Submit your manuscript here: https://www.dovepress.com/international-journal-of-nanomedicine-journal 